\title{
Panorama general de la gerencia de proyectos: una mirada en Panamá
}

\section{Overview of project management: a look at Panama}

\author{
Kaly Lore ${ }^{l}$, Luis Santos ${ }^{l}$, Patricio Bosquez ${ }^{l}$, Libia Batista ${ }^{1 *}$ \\ ${ }^{1}$ Doctorado en Gerencia, Universidad del Caribe
}

\begin{abstract}
Resumen La certificación a la gestión de proyectos representa para la organización y para los profesionales, una herramienta que agrega valor para producir valor. Este artículo presenta un enfoque general de la gestión de proyectos y su campo de interés está centrado en la importancia de una gestión de proyectos certificada, como garante del éxito en el impacto durante y después del proyecto, permitiendo que las organizaciones se encuentren en la vanguardia y generando satisfacción al dueño, al ejecutante y al cliente. Se hace una revisión que se desarrolla de la siguiente manera: 3.Gestión de proyectos. Una historia en evolución, 4. Estándares en la gestión de proyectos. Certificaciones y 5.Certificaciones a la gestión de proyectos en Panamá. Se hace especial énfasis a los aspectos positivos que conlleva una eficiente y eficaz gestión de proyectos, desde el impacto económico y social. Además, se presentan estadísticas sobre el tema en Panamá.
\end{abstract}

Palabras clave Certificación en proyectos, gerencia de proyecto, gestión, proyecto.

\begin{abstract}
Certification for project management represents for the organization and for professionals, a tool that adds value to produce value. This article presents a general approach to project management and the field of interest is focused on the importance of a certified project management, as a guarantor of the success in impact during and after the project, allowing organizations to be at the forefront and generating satisfaction to the owner, the performer and the client. A review is made that is developed as follows: 3. Project management. A story in evolution, 4. Standards in project management. Certifications and 5. Certifications to project management in Panama. Special emphasis is placed on the positive aspects of efficient and effective project management, from the economic and social impact. In addition, statistics on the subject are presented in Panama.
\end{abstract}

Keywords Project certification, project management, management, project.

*Corresponding author: libia.batista@utp.ac.pa

\section{Introducción}

Los proyectos han existido desde siempre, solo que con una noción diferente a la de hoy. El término de proyecto se aplica en la mayoría de las áreas del conocimiento y como concepto describen Nassir Sapag Chain y Reinaldo Sapag Chain "Un proyecto es la búsqueda de una solución inteligente al planteamiento de un problema tendiente a resolver, entre tantas, una necesidad humana" [1]. También merece destacar el concepto que emite la Organización de las Naciones Unidas (UNESCO), "Conjunto de actividades interrelacionadas que han de realizarse mediante una administración y unos recursos unificados, con el fin de lograr un objetivo específico en un plazo determinado..." [2]. En Panamá, el Sistema Nacional de Inversión Pública (SINIP), del Ministerio de Economía y Finanzas, como sistema regulador de la calidad de los proyectos de inversión pública, considera por su parte un proyecto como:

\begin{abstract}
"un conjunto de actividades planificadas y relacionadas entre sí, debidamente costeadas y programadas en el tiempo y en un espacio geográfico definido, con el propósito de solucionar problemas y/o necesidades y aprovechar oportunidades, contribuyendo con los objetivos de crecimiento y desarrollo socioeconómico sostenido del país" [3].
\end{abstract}

Por consiguiente, es importante enfatizar que un proyecto es una herramienta que de manera sistemática permite manejar variables de mucho valor como costo, tiempo, calidad, alcance, riesgo, todas en pro de un entregable a satisfacción del dueño, ejecutante y del cliente [4]. Un producto terminado que estará definido en base al problema a resolver, por ejemplo, si proviene de un proyecto de investigación, de un proyecto de inversión privada, de un proyecto de inversión social, otros. El éxito o fracaso de estos proyectos, cada vez más complejos, exigen más trabajo en equipo, sincronización de las tareas, más 
tecnologías, más sistematización, y es allí cuando se empieza a entender la necesidad de apostar por una eficiente y eficaz gestión de proyectos.

En este artículo se defiende la necesidad de la gestión de proyectos como garantía del progreso sostenible de las sociedades y de administrarse mejor los recursos para una empresa o institución. También, que representa una oportunidad de adquirir conocimiento para un mejor desempeño laboral y a la vez de superación profesional. Que es un ganar-ganar para una trilogía: satisfacción para las empresas, para el progreso social y personal. Además, se da a conocer lo que a nivel nacional e internacional se maneja sobre certificación en gestión de proyectos y su impacto.

\section{Metodología}

Esta investigación busca encontrar tendencias sobre la gestión de proyectos a nivel nacional e internacional. Para ello, se realizó un proceso investigativo tipo cualitativo-descriptivo, en el cual, se hizo uso de fuentes secundarias con el objetivo de brindar un soporte teórico a la temática de la gestión de proyectos y la certificación de gestión de proyectos. Además, este proceso investigativo busca especificar las características de la gestión propia en Panamá.

\section{Gestión de proyectos. Una historia en evolución}

Bajo este mundo globalizado, la subsistencia de las empresas y las respuestas a la sociedad por las instituciones de gobierno es cada día más difícil y especializado, de allí que la gestión de proyectos juega un rol importante en la rentabilidad económica y social. Se aprecia esta, cada día más dinámica y apuntando a la innovación, toda vez que representa una garantía para disminuir incertidumbre, identificar oportunidades, desarrollar mejores estrategias e identificar efectos o impactos que inciden en el éxito o fracaso de los proyectos.

La gestión de proyectos se proyecta como una herramienta de tipo gerencial, que permite a toda organización generadora de proyectos, potenciar las habilidades individuales y como equipo en la consecución eficiente y eficaz de lo planificado. "La Gestión de Proyectos, es la disciplina que tiene la capacidad de integrar de manera sistémica la planificación, la organización, la dirección, el control y la calidad en el manejo de las actividades asociadas a un proyecto" [5].

En su evolución, "el origen de la gestión de proyectos puede situarse a comienzos del siglo XX, considerándose la aparición de los primeros métodos" [6]. Como disciplina, su inicio está relacionada con la aparición del Diagrama de Gantt, en 1917; y para la década del 50 con la aparición del método PERT (Program Evaluation and Review Technique) y CPM (Critical Path Method), entra en otra fase y es cuando las organizaciones implementan el uso sistemático de las herramientas y técnicas de gestión de proyectos [6].

A través de ese proceso de mejora continua, en el desarrollo de sistemas complejos que requieren un equipo multidisciplinario, donde lo estable y lo predecible casi no existe, queda evidenciado para los años 60, el definir mejores métodos de organización y de trabajo y romper con esa constante de manejar proyectos con resultados improductivos, altos costos y de baja calidad [7]. De este escenario nace la necesidad de la profesionalización de la gestión de proyectos, una profesión garante del éxito de los proyectos. Por consiguiente, van surgiendo organizaciones con el propósito de que se hable y se escriba en el mismo idioma, en términos de gestión de proyectos. Es así como en el ámbito de la innovación, estas organizaciones establecen un sistema estandarizado fundamentado en conocimientos, metodologías, procesos y las prácticas para gestionar los proyectos con las mejores garantías en la toma de decisiones y calidad de los entregables. Mejores prácticas que afectan positivamente el impacto durante y después del proyecto.

\section{Estándares en la gestión de proyectos. Certificaciones}

La gestión de proyectos representa un interés a nivel mundial, desde las organizaciones y a nivel de profesionales, en ambos grupos, para hacerse más competitivos. En este contexto, las organizaciones buscan la certificación en gestión de proyectos y también, de profesionales certificados. Esto último, por ser una profesión que promete a las organizaciones las competencias y habilidades para reforzar el éxito de los proyectos. Demanda esta que va en crecimiento, ya que los proyectos cada vez son más costosos y con una exigencia alta en calidad. Este escenario es propicio para que las empresas requieran capital humano calificado. De allí, nace la necesidad de formación e induce al profesional a la búsqueda de la certificación profesional y a las organizaciones a certificarse.

En la actualidad existen varias certificaciones emitidas por diferentes asociaciones. Se puede mencionar las certificaciones del PMI, las de PRINCE2, las de AGILE, otras. Cada certificación en sí tienen varios tipos de certificación [8], [9]. En la tabla 1 se listan nueve organizaciones con su respectiva dirección electrónica. Se dedican estas a crear estándares para la gestión de los proyectos. 
Tabla 1. Organizaciones certificadoras

\begin{tabular}{|l|l|}
\hline \multicolumn{1}{|c|}{ Organización } & \multicolumn{1}{c|}{ Página web } \\
\hline $\begin{array}{l}\text { PMI, Project Management } \\
\text { Institute }\end{array}$ & www.pmi.org \\
\hline $\begin{array}{l}\text { APM, Association for } \\
\text { Project Management }\end{array}$ & www.apm.org.uk \\
\hline $\begin{array}{l}\text { AIPM, Australlian Institute } \\
\text { of Project Management }\end{array}$ & www.aipm.com.au \\
\hline $\begin{array}{l}\text { PMBN, Project Manag- } \\
\text { Benchmarking Network }\end{array}$ & www.pmbn.org \\
\hline $\begin{array}{l}\text { Swedish project management } \\
\text { society }\end{array}$ & www.projforum.se/en \\
\hline $\begin{array}{l}\text { PSA. Project management } \\
\text { Services for Australia }\end{array}$ & www.psaproject.com. \\
\hline $\begin{array}{l}\text { PMAJ, Project Management } \\
\text { Association of Japan }\end{array}$ & www.pmajor.jp/ENG \\
\hline $\begin{array}{l}\text { IPMA, International Project } \\
\text { Management Association }\end{array}$ & www.jpma.ch \\
\hline $\begin{array}{l}\text { ISO, International } \\
\text { Organization for } \\
\text { Standardization }\end{array}$ & www.iso.org \\
\hline
\end{tabular}

Fuente: “Análisis de la gestión de proyectos a nivel mundial” [10].

Estas certificadoras buscan ofrecer un estándar en la gestión de proyectos y lo hacen a través de un documento que acredita al que lo obtiene, disponer de un nivel de formación y experiencia en gerencia de proyectos, según la certificación obtenida. El Project Management Institute (PMI) define un estándar como "Un documento que proporciona, para uso común y repetido, reglas, pautas o características para actividades o sus resultados, orientado a lograr el óptimo grado de orden en un contexto determinado" [11].

\section{Certificaciones a la gestión de proyectos en Panamá}

La gestión de proyectos en Panamá desde la óptica pública y privada, se describe en esta investigación a través de la inversión de ambos sectores. En este sentido, la inversión gubernamental se muestra en las gráficas la y 1 b, mientras que la gráfica 2 refleja la inversión privada. En la figura 1a, se aprecia el aumento significativo de la inversión pública por los últimos cinco quinquenios.

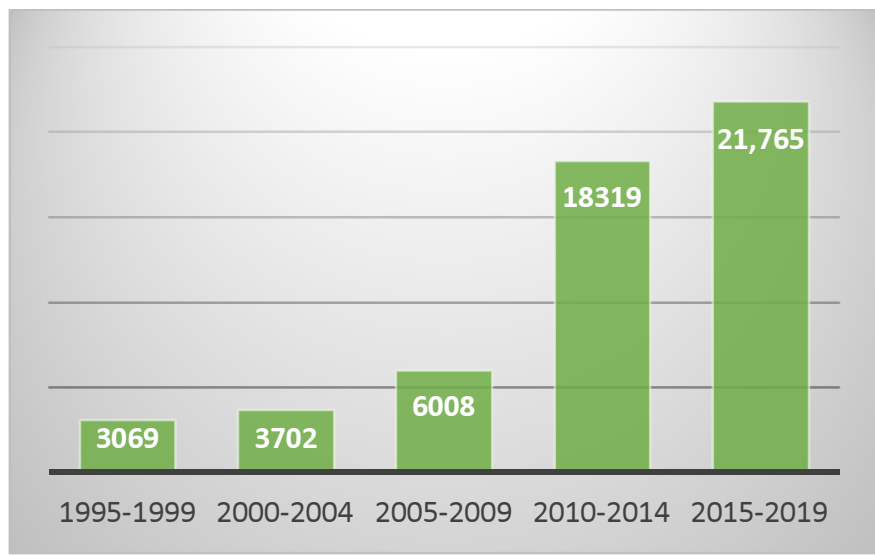

Gráfica 1a. Inversiones públicas del gobierno por quinquenio-En millones de USD.

Fuente: Datos del Ministerio de Economía y Finanzas [12].

La gráfica $1 \mathrm{~b}$ permite apreciar la inversión pública anual, para las vigencias 2014 a 2017.

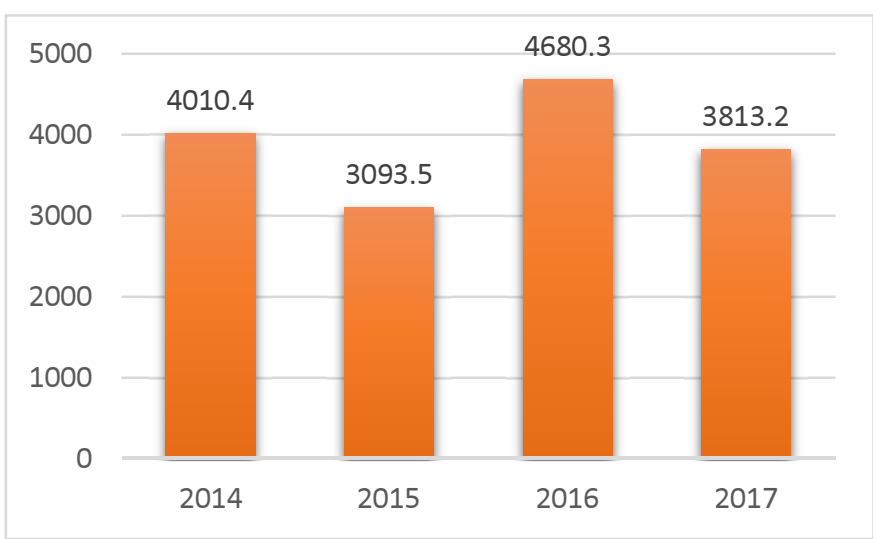

Gráfica 1b. Inversión pública (en millones de balboas).

Fuente: Elaboración propia con datos del Instituto Nacional de Estadística y Censo (INEC).

Desde el sector privado, la figura 2 revela la inversión anual desde 2014 a 2017.

Bajo este escenario de inversiones pública y privada en Panamá, se observa de una de las asociaciones formadores de profesionales en la dirección de proyectos a través de estándares y certificaciones reconocidas a nivel mundial, el 


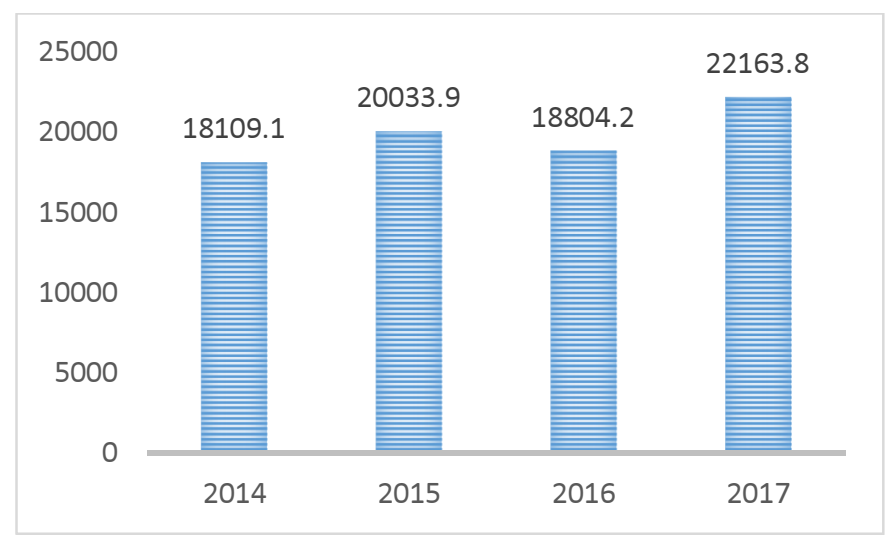

Figura 2. Inversión del sector privado (en millones de balboas). Fuente: Instituto Nacional de Estadística y Censo (INEC).

Project Management Institute (PMI), los miembros que han recibido dicha certificación en Panamá. En la tabla 1 se describe la cantidad de miembros por tipo de certificación.

Tabla 1. Miembros certificados en gestión de proyectos en el Capítulo de Panamá, según PMI-2019

\begin{tabular}{|c|c|}
\hline Total Members & 415 \\
\hline New members this year & 34 \\
\hline PMP $®$ Members & 216 \\
\hline CAPM $®$ Members & 16 \\
\hline PgMP® Members & 1 \\
\hline PMI-SP® Members & 3 \\
\hline PMI-RMP® Members & 7 \\
\hline PMI-ACP $®$ Members & 5 \\
\hline PfMP® Members & $\mathbf{0}$ \\
\hline PMI-PBA® Members & 2 \\
\hline
\end{tabular}

PMP/CAPM/PgMP/PMI-SP/PMI-RMP/PMI-ACP/PfMP/PMI-PBA are registered marks of the Project Management Institute, Inc.

Fuente: Project Management Institute (PMI) [13].

Otras certificaciones se han otorgado a través del Concejo de Certificación Profesional (CCP), quien es el organismo certificador IPMA en Panamá, y recibió sus credenciales de la sede en Zurich en el 2012 y a la fecha ha certificado a más de 500 profesionales panameños [14].

En cuanto a certificación a nivel de organizaciones, la Alcaldía de Panamá logra las certificaciones ISO 9001-2008 e IPMA DELTA-2017, con esto se convierte en el primer municipio de la región en obtener esta acreditación en la gestión de proyectos. "La Municipalidad de Panamá recibió su primera Certificación IPMA DELTA, de manos del Organismo Certificador Español de IPMA - Organismo de
Certificación en Dirección de Proyectos (ODCP) de AEIPROIPMA España [14], [15].

\section{Conclusiones}

De acuerdo al presente análisis de la gestión de proyectos en Panamá, existen entidades certificadoras como el PMI, Capítulo de Panamá; oficialmente constituido en el año 2004, el IPMA conformado desde el año 2012, si bien es cierto ambas certificadoras son de reciente creación, considerando que ya existía la necesidad de contar con las precitadas entidades certificadoras, no se formalizaban como Capítulos debido a falta de iniciativa.

A pesar de las grandes inversiones en megaproyectos en Panamá, no fueron consideradas contar con certificadoras, sin embargo, fue el impulso motor que permitió reconocer la necesidad de implementar en las condiciones y cláusulas de contratos, cumplir como requisitos entre otros, a fin de lograr con éxito los resultados esperados en cuanto a costos, tiempo y calidad.

Se aprecia certificaciones a panameños bajo el Project Management Institute (PMI) y del organismo certificador IPMA.

También merece la pena evaluar el impacto que ha tenido la certificación en proyectos por parte de la Alcaldía de Panamá.

Es importante valorar todas las certificadoras con sus respectivas metodologías para la gestión de proyectos en Panamá, y contrastar en una futura investigación, el contenido de sus especificaciones técnicas versus las competencias aplicables a las características del proyecto y así sustentar la certificación que mejor se adapta al país. Ese mismo estudio puede extrapolarse a nivel regional.

Otro estudio complementario en este tema de gestión de proyectos, lo constituye el evaluar la experiencia de profesionales certificados en aspectos como la influencia de la profesionalización en el impacto de los proyectos, además, su incidencia en términos de obtención de financiamiento.

\section{AGRADECIMIENTO}

Al Ingeniero José Reyes González, especialista en planificación estratégica y gestión de calidad, certificado en PMP de PMI e IPMA Nivel B por su colaboración, y contribuir con sus conocimientos y tiempo a nuestra investigación.

\section{REFERENCIAS}

[1] N. Sapag Chain and R. Sapag Chain, Preparación y evaluación de proyectos. .

[2] "Conceptos generales en la formulación y evaluación de proyectos - Documentos de Investigación - haydee131324," $2016 . \quad$ [Online]. Available: https://www.clubensayos.com/Negocios/Conceptos-generales- 
en-la-formulación-y-evaluación-de/3489840.html. [Accessed: 26-Mar-2019].

[3] Ministerio de Economía y Finanzas, "Normas y procedimientos del Sistema Nacional de Inversiones Públicas (SINIP)," Panamá, 2018.

[4] J. García Reyes, Gerencia de proyectos. Universidad de los Andes, 2013.

[5] R. A. Terrazas, "Modelo conceptual para la gestión de proyectos," Perspectivas, vol. 24, no. 1994-3733, pp. 165-188, 2009.

[6] Angel Nieva, "Innovación en la Gestión de Proyectos. Una historia en evolución." [Online]. Available: http://www.gedpro.com/Aldía/Noticias/Noticias_77.aspx. [Accessed: 27-Mar-2019].

[7] "Innovación en la Gestión de Proyectos. Una historia en evolución." [Online]. Available: http://www.gedpro.com/Aldía/Noticias/Noticias_77.aspx. [Accessed: 24-Mar-2019].

[8] "Certificación en proyectos | ¿Qué es y cómo conseguirla?" [Online]. Available: https://www.recursosenprojectmanagement.com/certificacionen-direccion-de-proyectos/. [Accessed: 29-Mar-2019].

[9] Carlos Álvarez, "Las 11 certificaciones top en gestión de proyectos del 2017 según CIO.COM," CIO.COM, 2017. [Online]. Available: https://www.linkedin.com/pulse/las-11certificaciones-top-en-gestión-de-proyectos-alvarez-g-pmp-. [Accessed: 27-Mar-2019].
[10] J. Nicolás and E. Reyes, "Palermo Business Review | No 12 | 2015 Análisis de la gestión de proyectos a nivel mundial."

[11] “¿Qué es un estándar?” [Online]. Available: http://americalatina.pmi.org/latam/pmbokguideandstandards/w hatisastandar.aspx. [Accessed: 05-Apr-2019].

[12] Ministerio de Economía y Finanzas, "Comparativo Quinquenal," 2018.

[13] "PMI Capítulo de Panamá." [Online]. Available: https://pmipanama.org/quienes-somos/que-es-pmi. [Accessed: 27-Mar2019].

[14] IPMA, “APGP2018.” [Online]. Available: https://www.apgpipma.org/. [Accessed: 09-Apr-2019].

[15] “Municipio de Panamá recibe certificaciones ISO 9001 e IPMA DELTAFuniber Blogs - FUNIBER." [Online]. Available: https://blogs.funiber.org/blog-proyectos/2017/07/17/funiberpanama-certificaciones-ipma-delta. [Accessed: 05-Apr-2019].

[16] I. 14 Project Management Institute, Guía de los fundamentos para la dirección de proyectos (guía del PMBOK®), no. 978-162825-9-1. 2013. 